\title{
Effect of Blade Geometry on the Aerodynamic Loads Produced by Vertical-Axis Wind Turbines
}

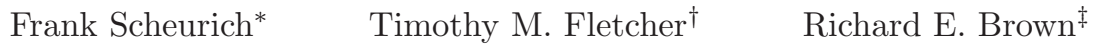

\begin{abstract}
Accurate aerodynamic modelling of vertical-axis wind turbines poses a significant challenge. The rotation of the turbine induces large variations in the angle of attack of its blades that can manifest as dynamic stall. In addition, interactions between the blades of the turbine and the wake that they produce can result in impulsive changes to the aerodynamic loading. The Vorticity Transport Model has been used to simulate the aerodynamic performance and wake dynamics of three different vertical-axis wind turbine configurations. It is known that vertical-axis turbines with either straight or curved blades deliver torque to their shaft that fluctuates at the blade passage frequency of the rotor. In contrast, a turbine with helically twisted blades delivers a relatively steady torque to the shaft. In the present paper, the interactions between helically twisted blades and the vortices within their wake are shown to result in localized perturbations to the aerodynamic loading on the rotor that can disrupt the otherwise relatively smooth power output that is predicted by simplistic aerodynamic tools that do not model the wake to sufficient fidelity. Furthermore, vertical-axis wind turbines with curved blades are shown to be somewhat more susceptible to local dynamic stall than turbines with straight blades.
\end{abstract}

Keywords: Vertical-Axis Wind Turbine; Blade-Wake Interaction; Dynamic Stall; Numerical Simulation; Vorticity Transport Model

* School of Engineering, University of Glasgow, UK, f.scheurich@aero.gla.ac.uk (corresponding author)

$\dagger$ School of Engineering, University of Glasgow, UK

$\ddagger$ Department of Mechanical Engineering, University of Strathclyde, Glasgow, UK

Based on Scheurich F, Fletcher TM, Brown RE. The Influence of Blade Curvature and Helical Blade Twist on the Performance of a Vertical-Axis Wind Turbine. AIAA-2010-1579. Presented at the 48th AIAA Aerospace Sciences Meeting, Orlando, USA, 2010. 


\section{Introduction}

The number of wind turbines deployed around the globe has increased significantly in recent years in an attempt to curb emissions of carbon dioxide and other pollutants. The wind turbines that are currently operating in wind farms feature, almost exclusively, a threebladed rotor with a horizontal-axis configuration. In recent years, however, there has been a resurgence in the development of both large-scale and small-scale vertical-axis wind turbines. The development of large-scale vertical-axis wind turbines is mainly a response to a plateau in the improvement of the aerodynamic performance of horizontal-axis wind turbines. Indeed, the UK Energy Technologies Institute is conducting the Novel Offshore Vertical Axis (NOVA) project [1] with the aim of installing a prototype large offshore vertical-axis wind turbine by the year 2020. The study hopes to demonstrate that such devices have improved stability and simpler maintenance access compared to horizontal-axis designs. The research into smallscale vertical-axis wind turbines is motivated largely by a perceived demand for decentralized electricity generation within cities and rural communities.

Vertical-axis wind turbines are, by design, insensitive to the wind direction if the wind is perpendicular to the axis of rotation, and therefore do not require a yaw control system. Horizontal-axis wind turbines, in contrast, have to be rotated in order to track changes in wind direction. Several authors, including Mertens [2], have suggested that vertical-axis wind turbines thus offer a distinct advantage over those with horizontal-axis design when operating in conditions that are typical of urban environments.

A vertical-axis wind turbine is called a Darrieus turbine when it is driven by aerodynamic lift. The concept was patented in the United States by Georges Darrieus [3] in 1931. The patent includes both straight- and curved-bladed configurations in which the blades are supported using plates located at the top and the bottom of the turbine to provide structural strength. By curving the blades to yield the so-called troposkien shape, which is equivalent to the shape of a spinning rope that is constrained at its ends, the high bending moments that are encountered on straight-bladed turbines can, to some extent, be alleviated. Both 
straight-bladed and curved-bladed vertical-axis wind turbines suffer, however, from a marked azimuthal variation in the aerodynamic loading on their blades. These variations in loading manifest as oscillations in the torque and power that is output from the turbine. The oscillating torque that is developed by straight- and curved-bladed turbines contributes significantly to the vibration that is transmitted to the tower and the foundations of the system. In addition, the oscillations in the loading on the blades can lead to increased fatigue of the rotor structure. For these reasons, several commercial vertical-axis wind turbines feature a design that incorporates blades that are twisted helically around the rotational axis of the turbine. Although its blades individually still experience cyclic variations in aerodynamic loading, the torque, and consequently the power output, of a vertical-axis wind turbine with helically twisted blades is relatively steady, thus alleviating the stress on the turbine system, reducing the vibration transmitted into its supporting structure, and thereby increasing the design life of the turbine.

The accurate aerodynamic simulation of vertical-axis wind turbines poses a significant challenge. During operation, the angle of attack of the blades varies cyclically with azimuth. Dynamic stall can occur at low tip speed ratios when the angle of attack of the blades transiently exceeds the static stall angle of their aerofoil sections. In addition, the interactions between each of the turbine blades and either their own wake, or the vorticity that is developed downwind of the other blades, create an impulsive component to the aerodynamic loading on the blades. These interactions are notoriously difficult to predict accurately in terms of their position and strength. Indeed, Klimas [4] considered the interaction between the blades of the turbine and the wake that is produced by the rotor to be one of the most critical problems in the numerical modelling of the aerodynamics of vertical-axis wind turbines. In the present paper, a computational model is used to compare the aerodynamic performance of a turbine with straight blades to that of a second turbine with curved blades, and to that of a third turbine with blades that are helically twisted around the rotational axis. The model that is used for the aerodynamic simulation of the different vertical-axis wind turbine configurations is capable of capturing the interactions between the blades of the turbine and the wake that is developed by the rotor and thus allows a detailed analysis of the influence of the blade-wake 
interactions on the distribution of the aerodynamic loading along the blades of the turbine to be performed.

\section{Computational Aerodynamics}

The aerodynamic performance and wake dynamics of three vertical-axis wind turbine configurations have been simulated using the Vorticity Transport Model (VTM) developed by Brown [5], and extended by Brown and Line [6]. The VTM enables the simulation of wind turbine aerodynamics and performance by providing a high-fidelity representation of the dynamics of the wake that is generated by the turbine rotor. In contrast to more conventional computational fluid dynamics techniques in which the flow variables are pressure, velocity and density, the VTM is based on the vorticity-velocity form of the unsteady incompressible Navier-Stokes equation

$$
\frac{\partial}{\partial t} \omega+u \cdot \nabla \omega-\omega \cdot \nabla u=S+\nu \nabla^{2} \omega
$$

The advection, stretching, and diffusion terms within Equation (1) describe the changes in the vorticity field, $\omega$, with time at any point in space, as a function of the velocity field, $u$, and the kinematic viscosity, $\nu$. The physical condition that vorticity may neither be created nor destroyed within the flow, and thus may only be created at the solid surfaces that are immersed within the fluid, is accounted for using the vorticity source term, $S$. The vorticity source term is determined as the sum of the temporal and spatial variations in the bound vorticity, $\omega_{b}$, on the turbine blades, so that

$$
S=-\frac{d}{d t} \omega_{b}+u_{b} \nabla \cdot \omega_{b}
$$

In the VTM, Equation (1) is discretized in finite-volume form using a structured Cartesian mesh within a domain that encloses the turbine rotor, and then advanced through time using an operator-splitting technique. The numerical diffusion of vorticity within the flow field surrounding the wind turbine is kept at a very low level by using a Riemann problem technique based on the Weighted Average Flux method developed by Toro [7] to advance the vorticity convection term in Equation (1) through time. This approach permits many rotor revolutions 
to be captured without significant spatial smearing of the wake structure and at a very low computational cost compared with those techniques that are based on the pressure-velocitydensity formulation of the Navier-Stokes equations. Dissipation of the wake does still occur, however, through the proper physical process of natural vortical instability.

The bound vorticity distribution on the blades of the rotor is modelled using an extension of lifting-line theory. The lifting-line approach has been appropriately modified by the use of twodimensional experimental data in order to represent the real performance of any given aerofoil. The effect of dynamic stall on the aerodynamic performance of an aerofoil is accounted for by using a semi-empirical dynamic stall model that is based on the model that was proposed by Leishman and Beddoes $[8,9]$. The Leishman-Beddoes model was originally developed to simulate the effect of dynamic stall on the blades of a helicopter by deriving the calculation of the non-linear aerodynamic coefficients from Kirchhoff theory [10]. Gupta and Leishman [11] demonstrated that a modified version of the original Leishman-Beddoes model can be used to represent the dynamic stall of aerofoils that are comparable to those used for horizontal-axis wind turbines. Angell, Musgrove and Galbraith [12] investigated the occurrence of dynamic stall on an aerofoil that was operated to mimic the changes in angle of attack that the aerofoil would experience on a vertical-axis wind turbine, and revealed that the tangential force coefficient can become negative under deep stall conditions. This observation conflicts with the Kirchhoff theory since the calculation of the tangential force coefficient, based on this theory, will yield values that are greater than or equal to zero for every flow state. Sheng, Galbraith and Coton [13] suggested, therefore, a modification for the modelling of the tangential force coefficient by inclusion of an additional parameter into the model that accounts for negative tangential forces at low Mach numbers in deep stall. The Leishman-Beddoes-type dynamic stall model that is implemented in the VTM includes this modification for the calculation of the tangential force coefficient, and has also further been modified as suggested by Niven and Galbraith [14], to account for vortex inception at low Mach numbers.

Figure 1 shows the variation of the predicted normal and tangential force coefficients of a NACA 0015 aerofoil in a dynamic stall test in comparison to experimental measurements that 
were made by Angell, Musgrove and Galbraith [12] for a Reynolds number of 800,000, a Mach number of 0.064 and a reduced frequency of 0.05. Results are presented in Figure 1 for a test in which the amplitude of the oscillation of the angle of attack was $21.80^{\circ}$ in order to show the effect of deep dynamic stall on the aerodynamic performance of the aerofoil. The geometric angle of attack of the blade of a rotating vertical-axis wind turbine, $\alpha$, is a function only of the tip speed ratio, $\lambda$, and the azimuth angle, $\psi$, such that

$$
\alpha=\arctan \left(\frac{\sin \psi}{\lambda+\cos \psi}\right)
$$

Thus, the test yields conditions that are equivalent to those experienced by the blades of a vertical-axis wind turbine when operating at a tip speed ratio of 2.70. Although some minor discrepancies between the experimental measurements and the VTM-predictions are apparent, the variations with angle of attack of the VMT-predicted normal and tangential force coefficients agree, overall, reasonably well with the experimental measurements in terms of shape and magnitude of the hysteresis loops, and therefore provide confidence that the effect of dynamic stall is satisfactorily accounted for in the VTM.

The VTM was originally developed for simulating the flow field surrounding helicopters, but is an aerodynamic tool that is applicable also to the study of wind turbine rotors. Indeed, Fletcher et al. [15] validated VTM predictions of the performance of a horizontal-axis wind turbine against experimental data that was published by Hand et al. [16], and compared VTM predictions of wind turbine performance to those of more conventional RANS methods. Fletcher and Brown [17] have also used the VTM successfully to investigate the aerodynamic interaction between horizontal-axis wind turbines within a wind farm. Furthermore, Scheurich, Fletcher and Brown [18] have validated the VTM predictions against experimental measurements of the performance of a straight-bladed vertical-axis wind turbine that were made by Strickland, Smith and Sun [19]. 


\section{Turbine Model}

The geometry of each of the three vertical-axis wind turbines that are investigated in this paper is illustrated in Figure 2. The blades of each turbine are separated by $120^{\circ}$ azimuth. Starting from the straight-bladed configuration, 'a', shown in Figure 2(a), the radial location of each blade section was displaced using a hyperbolic cosine distribution in order to yield the troposkien shape of configuration 'b', as shown in Figure 2(b). Configuration 'c', shown in Figure 2(c), was then obtained by applying helical blade twist around the rotor axis. The blades of the straight- and curved-bladed configuration, and each individual section of the blades of the helically twisted configuration, are designed to have zero geometric pitch angle. In other words, the chord of each blade section is tangential to the local segment of the circle that is described by the trajectory of the blades. The maximum radius, $R$, of each rotor is at the mid-span of the reference blade ('blade 1') of the turbine, and is identical for each of the three different configurations. This radius is used as the reference radius of the rotor when presenting non-dimensional data for the performance of the rotor. The tip speed ratio, $\lambda$, is defined as the ratio between the circumferential velocity at the mid-span of the blade, $\Omega R$, and the wind speed, $V_{\infty}$. The geometric properties of the three turbines that were investigated are listed in Table 1, whereas the key parameters of the straight-bladed turbine, which is treated throughout this paper as the baseline configuration against which the other turbines are compared, are summarized in Table 2. The orientation of the blades and specification of the azimuth angle of the turbine with respect to the mid-span of blade 1 is shown in Figure 3. The simulations were carried out under the assumption that the turbine is operated in steady, uniform wind conditions. This was done in order to isolate the unsteady blade aerodynamic loading that is caused simply by the rotation of the turbine from the unsteady loading that would be induced by non-uniform and unsteady inflow in the real operating environment. An exploration of the aerodynamic characteristics of the turbines has been conducted over their entire range of operational tip speed ratios. As the tip speed ratio is varied, the relative contribution of dynamic stall, blade-vortex interaction and other wake related effects on the 
loading on the turbine blades does change. In this paper, however, given constraints of space, only those results for a tip speed ratio of five, in other words for a tip speed ratio somewhat near the mid-range of operationally-relevant tip speed ratios, are presented. These results show the clearest independent manifestation of all the physical effects that govern the aerodynamic behaviour of the turbine configurations that are studied in the present work.

\section{Verification of Aerodynamic Predictions}

Before comparing the performance of the three different configurations of vertical-axis wind turbine that were defined in the previous section, it is essential to establish first that the requisite fluid dynamic features within the flow surrounding the turbine rotor, and hence the aerodynamic loads on the blades of the rotor, are correctly simulated by the VTM. Figure 4 shows the VTM-predicted non-dimensional normal and tangential forces generated by the vertical-axis wind turbine with two straight blades, operating at a tip speed ratio of five, for which experimental measurements were obtained by Strickland, Smith and Sun [19].

The local blade velocity varies cyclically with azimuth due to the sinusoidal variation in the component of free stream velocity relative to the blade chord. On the windward side of the turbine, at $90^{\circ}$ azimuth, the free stream velocity vector is orthogonal to the blade chord, resulting in a high angle of attack and an associated peak in the blade loading. As the blade passes towards the leeward side of the turbine, between $180^{\circ}$ and $360^{\circ}$ azimuth, the flow that is induced by the wake of the turbine results in a reduction in the aerodynamic loading on the blades. Very satisfactory agreement between experimental measurements and VTM predictions for both the non-dimensional normal and tangential force is observed for the entire azimuth as shown in Figures 4(a) and 4(b). A more detailed comparison between the VTM-predicted loading on this particular turbine and the equivalent experimental measurements performed by Strickland, Smith and Sun [19] is given by Scheurich, Fletcher and Brown [18].

The good agreement between the VTM-predicted forces and the experimental measurements provides confidence in the ability of the method to simulate accurately the aerodynamic loading on the blades of the vertical-axis wind turbines that are analysed in the present work. 


\section{Cyclic Aerodynamic Loading}

The variation with azimuth of the aerodynamic loading on the blades of each of the three different vertical-axis wind turbine configurations features a cyclic component, which is described in detail in the present section, and an impulsive component which is examined in more detail in Section 6 of this paper. The cyclic variation of the blade loading with azimuth is due to the sinusoidal variation of the component of free stream velocity with respect to the blade chord, and is characterized by a peak in the aerodynamic loading on the blade close to $90^{\circ}$ azimuth, i.e. in the upwind part of the rotor revolution. On the leeward side of the turbine, between $180^{\circ}$ and $360^{\circ}$ azimuth, however, the blades interact with the vortices in the wake of the turbine to produce a highly impulsive component to the loads on the system.

Figure 5(a) shows the variation with azimuth of the angle of attack at four spanwise locations along the reference blade of the straight-bladed turbine, as defined in Figure 5(b). The variation with azimuth of the sectional non-dimensional normal and tangential forces is presented in Figures 5(c) and 5(d).

A peak occurs in the variation of the angle of attack, and consequently in the blade aerodynamic loading close to $90^{\circ}$ azimuth. The behaviour of this turbine is thus very similar to that of the two-bladed turbine for which the blade loading was presented in Figure 4. Figure 5(a) illustrates that the angle of attack close to the blade tip $(z / b=0.125$ and $z / b=0.045)$ is lower than that nearer to the mid-span of the blade. This effect is caused by the finite span of the blade and thus the influence of the tip vortices in inducing a strong component of flow, normal to the blade chord, near the tips of the rotor blades. On the leeward side of the turbine, large, transient perturbations in the angle of attack are induced near to the tip of the blade. These impulsive perturbations are caused by interactions between the blade and the vortices that are trailed and shed from the blades in previous rotor revolutions, as discussed in more detail in Section 6 of this paper.

Figure 6(a) shows the variation with azimuth of the angle of attack at four spanwise locations along the reference blade of the curved-bladed turbine, as defined in Figure 6(b). The 
variation with azimuth of the sectional non-dimensional normal and tangential forces is presented in Figures 6(c) and 6(d).

Figure 6 shows the cyclic variation of the angle of attack with azimuth for the curved-bladed turbine to resemble that of the straight-bladed turbine, with a similar peak in the blade loading close to $90^{\circ}$ azimuth. The variation of the angle of attack along the blade span differs from that observed for the straight-bladed turbine, however, as the curvature of the blades results in a reduced radius, and, consequently, close to the blade tip, in a reduced circumferential component of velocity relative to the free stream. The amplitude of the oscillation of the local angle of attack close to the tip is thus significantly greater than that at the mid-span of the blade. Indeed, Figure 6(a) shows that, near the tip of the blade, the angle of attack increases periodically beyond the static stall angle for the NACA 0015 aerofoil. This indicates that the region near to the tips of the blades of the curved-bladed turbine configuration is subject to dynamic stall, even at tip speed ratios that might be considered to be in the mid-region of the operating envelope. Figure 6 illustrates that the blades of this turbine are also subject to large, transient perturbations to the angle of attack, and thus to the blade loading, close to the tip of the blade when the blades pass through the wake that is produced by the turbine. These perturbations have a similar origin to those shown in Figure 5 for the straight-bladed turbine, but are confined to those sections nearest to the tips of the blade.

Figure 7(a) shows the variation with azimuth of the angle of attack at four spanwise locations along the reference blade of the turbine with helically twisted blades, as defined in Figure 7(b). The variation with azimuth of the sectional non-dimensional normal and tangential forces is presented in Figures $7(\mathrm{c})$ and $7(\mathrm{~d})$.

Figure 7(a) indicates that, near to its tips, the angle of attack of the reference blade of the turbine with helically twisted blades also periodically exceeds the static stall angle of the aerofoil. This is, again, a consequence of the reduced circumferential component of velocity relative to the free stream near to the tips of the blades, which is associated with the reduced diameter of the rotor near the blade tips when compared to that at the mid-span of the blade. The prime consequence, however, of the helical twist that is applied to the turbine with 
configuration ' $\mathrm{c}$ ' is the phase lag in the angle of attack and, thus, in the loading along the length of the blades. The progressive phase shift in the variation of the angle of attack, and consequently in the blade loading along the length of the blade is a result of the distribution of blade area over an azimuthal sector of the turbine, as seen in Figure 2(c). In the downwind part of the revolution of the blade, between $180^{\circ}-360^{\circ}$ azimuth, impulsive perturbations in the variation of the angle of attack and the blade loading, although most marked near the tip of the blade, are also observed further inboard. This is indicative of a broader distribution of interactions between the blades and the vortices within the wake of the turbine with helically twisted blades than on the other two turbine configurations described in this paper. The sectional normal forces close to the blade tip are smaller than those experienced at the mid-span of the blade. Interestingly, the sectional tangential forces that are generated on the outboard part of the blade are slightly greater than the loads that are generated at the mid-span of the blade, however. The reduction in the local velocity that is experienced by the outboard portion of the blade that is due to its smaller radius might lead one to the conclusion that the sectional tangential force close to the blade tip should also be lower than that at mid-span of the blade. This effect is offset, however, by the higher angle of attack close to the tip of the blade which results in local tangential forces that are similar or even greater in magnitude than those experienced at the centre of the blade. The importance of this observation is discussed further in the Section 7 of this paper, where the variation with azimuth of the torque that is produced by the different turbine configurations is compared.

\section{Impulsive Blade Loading due to Blade-Wake-Interaction}

The particular advantage of the Vorticity Transport Model is that it allows the wake of the turbine to be captured without significant spatial smearing of its structure during the many revolutions of the rotor in which the wake remains in close proximity to the blades. This renders the model particularly adept at resolving in detail the interaction between the blades of the turbine and the wake.

A three-dimensional representation of the wake that is produced by each of the three differ- 
ent turbine configurations is presented in Figure 8. Each wake is visualized as a surface within the flow on which the vorticity has constant magnitude. Figures 8(a), 8(c) and 8(e) illustrate the overall wake structure that is developed by each of the three turbine configurations, whilst, in order to reveal the complexity of the vortical structure within the wake, in Figures 8(b), 8(d) and 8(f) separate shades are used to show the vorticity that is created by each of the three blades of the turbines. The curved-bladed turbine produces a wake that is somewhat more compact due to the smaller radius of the outboard portion of the blades compared with the wake that is produced by the straight-bladed and helically twisted configuration.

Figures $9(\mathrm{a}), 9(\mathrm{~b})$ and $9(\mathrm{c})$ show the vorticity distribution on a plane through the centre of each of the three turbine configurations. This plane is oriented perpendicular to the ground and is aligned with the wind direction. The vorticity distribution is depicted at the instant of time when blade 1 is located at $270^{\circ}$ azimuth. The flow field is represented using contours of the component of vorticity perpendicular to the plane. The dark rendering corresponds to vorticity with a clockwise sense, and the light rendering to vorticity with a counter-clockwise sense of rotation. Figures $9(\mathrm{a})$ and 9 (b) illustrate that the non-twisted blades of the straightand curved-bladed turbines result in a relatively symmetric distribution of vorticity in the wake downstream of the turbine. In contrast, the flow field of the turbine with helically twisted blades is characterised by a strong asymmetry in the distribution of vorticity between the upper and lower halves of the turbine, as is shown in Figure 9(c). It is apparent that their mutual induction causes the vortices that are produced by the blades of the turbine to convect towards the centreline of the rotor. The visualisation of the wake allows the origins to be identified of the localized impulsive perturbations that were observed in the variation of the angle of attack and the aerodynamic loading on the blades of each of the three turbine configurations. Indeed, the large, transient perturbations in the variation of the angle of attack and the blade loading of the straight-bladed turbine that were observed in Figure 5 can be related directly to the interaction between the reference blade and a region of vorticity in the wake, as depicted in Figure 9(a). This region of vorticity consists of the vortices that were generated by the blades in previous rotor revolutions. It can also be seen why, in the absence of significant 
interactions with the tip vortices, the angle of attack, and consequently the blade loading, at the mid-span of the blade $(z / b=0.5)$ does not exhibit the same impulsive perturbations that are observed at blade sections further outboard. This observation is important as it implies that an appropriate three-dimensional representation of the wake is an essential component of any model that is designed to capture accurately the aerodynamics of vertical-axis wind turbines.

The vorticity distribution that is produced in the wake of the turbine with curved blades is shown in Figure 9(b). Compared to the extent of the blade-wake interactions that occur in the straight-bladed turbine system, the blade-wake interactions within the curved-bladed turbine system can be seen to be confined to a smaller portion of the blade near to the tip. This observation is consistent with Figure 6 which showed the transient perturbations in the angle of attack and the aerodynamic loading to be confined to a much smaller region near to the very tip of the blade than was the case with the straight-bladed turbine.

The distribution of vorticity in the wake that is produced by the turbine with helically twisted blades is shown in Figure 9(c), and is reproduced again in Figure 10 with the rotor in three different azimuthal orientations to expose the migration along the length of the blade, as the turbine rotates, of the regions of most intense blade-vortex interaction. In Figures 10(a), 10(b) and 10(c), the mid-span of the reference blade is located at $300^{\circ}, 320^{\circ}$ and $340^{\circ}$ azimuth, respectively. At $300^{\circ}$ azimuth, principal interactions with the vortices within the wake occur at section $z / b=0.25$ of blade 1 , but as the turbine rotates, these interactions move outboard along the blade. This migration is the origin of the broad distribution along the span of the blades of the impulsive perturbations in the variation of the angle of attack and the blade loading that are observed in Figure 7.

\section{Variation in Torque Coefficient and Rotor Forces}

The variation in torque coefficient of the turbine is analysed, at first, by investigating the sectional torque at different spanwise locations. The analysis of the sectional torque, which is the product of the sectional tangential force and the sectional radius, allows the contribution 
of the torque that is produced by a blade section to the torque that is produced by the entire turbine to be quantified. Figure 11 shows the sectional non-dimensional torque, $Q^{*}$, at four spanwise locations of the reference blades that are defined in Figures 5(b), 6(b) and 7(b). The angle of attack, and consequently the local tangential force coefficient, for those sections close to the tip of the blades of the turbine with helically twisted blades is higher when compared with those sections nearer to the mid-span of the blade. This can be understood once it is realised that blade curvature results in a smaller radius for the sections that are located close to the blade tip in comparison to those that are located towards the mid-span of the blade. The reduced radius of the sections close to the blade tip also results in a lower local velocity at those sections, however. Indeed, it is the combination of a lower local velocity and a higher force coefficient due to a higher angle of attack that results in a relatively uniform distribution of torque being developed along the length of the blades of the helically twisted turbine if the blades are designed so that blade curvature and helical blade twist are properly matched.

The azimuthal variation of the loading on the blades yields significant unsteadiness in the torque and hence the power that is produced by a vertical-axis wind turbine if its blades are not twisted. The introduction of helical blade twist together with blade curvature can reduce significantly the oscillations in torque output from the turbine. This is demonstrated in Figure 12, where the variation of the unsteady component of the torque coefficient, $\Delta C_{Q}$, for one turbine revolution is presented for the three different turbine configurations. Although the key rotor parameters of the three different wind turbines are identical, the difference in their aerodynamic design leads, naturally, to different absolute values of their torque coefficients even if they are operated at the same tip speed ratio. In order to make a rigorous comparison between the turbine configurations, the variation of the torque coefficient from the mean torque coefficient is compared in Figure 12. The mean torque coefficients for the turbines with straight, curved and helically twisted blades are summarised in Table 3.

The straight- and curved-bladed turbines exhibit significant unsteadiness in torque coefficient over the course of a single turbine revolution. The oscillations in the torque coefficients of the straight- and curved-bladed turbines have three coherent peaks close to $90^{\circ}, 210^{\circ}$ and 
$330^{\circ}$ azimuth; this reflects the location of the peak in the variation of the torque of each single blade close to $90^{\circ}$ azimuth, as shown in Figures 11(a) and 11(b). The torque coefficient of the turbine with helically twisted blades, in contrast, is relatively steady. Despite each blade section of the helically twisted blade experiencing fluctuations in blade loading with azimuth, the more uniform distribution of its blade area around the azimuth of the turbine results in a significant reduction in the unsteadiness of the torque that is transmitted through the shaft, when compared to the turbines with untwisted blades. The azimuthal variation of the torque coefficient of the turbine with helically twisted blades is not entirely smooth, however. A residual unsteadiness in the torque output of the turbine results partially from the incomplete overlap of the three blades, but also from the perturbations to the blade loading that are caused by the blade-vortex interactions shown in Section 6 of this paper.

The mean forces that act on the rotor for the three configurations that were investigated are summarised in Table 3. The axial rotor force on the straight-bladed configuration is zero, whereas the curvature of the blades on the helically twisted and curved-bladed turbine results in very low, but nevertheless non-zero, values for the axial rotor force. The magnitude of the axial rotor force is somewhat negligible, however, compared with the side force, $F_{Y, \text { Rotor }}^{*}$, and

the drag force, $F_{X, \text { Rotor }}^{*}$, of the rotor. The oscillations that are observed for the torque coefficient of the straight- and curved-bladed configuration also manifest in oscillations of the rotor forces, as shown in Figures 13(a) and 13(b). In contrast, the oscillations in the side force, $F_{Y, R o t o r}^{*}$, and the drag force, $F_{X, \text { Rotor }}^{*}$, of the rotor with the helically twisted blades are significantly reduced, as illustrated in Figure 13(c). The reduction in the oscillations of the rotor forces on the helically twisted configuration is very beneficial since it might reduce vibration and material fatigue on the tower and foundation of the turbine thus enhancing its life span.

\section{Conclusion}

The aerodynamic performance and wake dynamics of three vertical-axis wind turbines, one with straight blades, another with curved blades and a third with a helically twisted blade configuration, have been investigated using the Vorticity Transport Model (VTM). The VTM 
explicitly conserves the vorticity within the flow field surrounding the turbine, thus enabling the influence on turbine performance of the structure and evolution of the vortical wake that is induced by the rotor to be analyzed in detail. Vertical-axis wind turbines with either straight or curved blades are known to suffer from blade loads that vary considerably with azimuth; this results in a torque output that contains a substantial oscillatory component at the blade passage frequency of the turbine. The variations in the blade loading can fatigue the rotor structure and reduce the design life of the turbine. By comparison, a turbine with helically twisted blades produces a relatively steady torque. Despite the helically twisted blades still individually experiencing oscillations in blade loading with azimuth, a relatively steady force distribution for the entire turbine is achieved through an almost uniform distribution of blade area around the azimuth. The torque output of the turbine with helically twisted blades is not entirely smooth, however, as the mutual interactions between the blades and the vortices within the wake that is developed by the rotor do still result in localized impulsive perturbations to the aerodynamic loading on the blades.

Blade curvature results in a reduced circumferential velocity relative to the free stream velocity at the outboard region of the blade when compared to that at the mid-span of the blade. This is because the effective radius of the blade sections is smaller near to the blade tips than at the mid-span of the blade. The outboard regions of the blades of the curved-bladed configuration therefore experience oscillations in angle of attack that are of larger amplitude than those at the mid-span of the blade. The angle of attack can exceed, at least transiently, the range in which the lift coefficient varies linearly with angle of attack, and can thus manifest as dynamic stall at higher tip speed ratios than for the straight-bladed configuration.

The analysis that is presented in this paper reveals that interactions between the blades of a vertical-axis wind turbine and the wake that they produce can be captured if an appropriate model for the three-dimensional wake is used in the aerodynamic prediction. It is, therefore, concluded that such a model is a crucial component of any numerical scheme that will be able to predict accurately the aerodynamic loading on the blades of vertical-axis wind turbines. 


\section{References}

1. ETI. Energy Technologies Institute unveils first projects to benefit from £1.1 billion initiative. ETI, Press Release: Loughborough, UK, 13 January 2009.

2. Mertens, S. Wind Energy in the Built Environment. Multi-Science Publishing Co. Ltd, Brentwood, UK, 2006.

3. Darrieus, G. J. M. Turbine having its rotating shaft transverse to the flow of the current. US Patent 1,835,018. December 1931.

4. Klimas, P. C. Darrieus rotor aerodynamics. Transactions of the ASME. Journal of Solar Energy Engineering. 1982, 104, 102-105.

5. Brown, R. E. Rotor wake modelling for flight dynamic simulation of helicopters. AIAA Journal 2000, 38, 57-63.

6. Brown, R. E. and Line, A. J. Efficient high-resolution wake modelling using the vorticity transport equation. AIAA Journal 2005, 43, 1434-1443.

7. Toro, E. A weighted average flux method for hyperbolic conservation laws. In Proceedings of the Royal Society of London, Series A: Mathematical and Physical Sciences, 1981, 423, 401418.

8. Leishman, J. G. and Beddoes, T. S. A semi-empirical model for dynamic stall. Journal of the American Helicopter Society, 1989, 34, 3-17.

9. Beddoes, T. S. A third generation model for unsteady aerodynamics and dynamic stall. Westland Helicopters Ltd., RP-908, 1993.

10. Thwaites, B. Incompressible Aerodynamics. Oxford University Press, UK, 1960.

11. Gupta, S. and Leishman, J. G. Dynamic stall modelling of the S809 aerofoil and comparison with experiments. Wind Energy, 2006, 9, 521-547.

12. Angell, R. K., Musgrove, P. J. and Galbraith, R.A.McD. Collected data for tests on a NACA0015 - volume III: pressure data relevant to the study of large scale vertical axis wind 
turbines. Department of Aerospace Engineering, University of Glasgow, Glasgow, UK, G.U. AERO REPORT 8803, 1988.

13. Sheng W., Galbraith RAMcD, Coton, FN. A modified dynamic stall model for low Mach numbers. Journal of Solar Energy Engineering 2008; 130: 031013/1-10.

14. Niven, A. J. and Galbraith, R.A.McD. Modelling dynamic stall vortex inception at low Mach numbers. Aeronautical Journal, 1997 101, 67-76.

15. Fletcher, T. M., Brown, R. E., Kim, D. H. and Kwon, O. J. Predicting wind turbine blade loads using vorticity transport and RANS methodologies. In Proceedings of the European Wind Energy Conference, Marseille, France, March 2009.

16. Hand, M. M., Simms, D. A., Fingersh, L. J., Jager, D. W., Cotrell, J. R., Schreck, S. and Larwood, S. M. Unsteady aerodynamics experiment phase VI: wind tunnel test configurations and available data campaigns. NREL Technical Report NREL/TP-500-29955, 2001.

17. Fletcher, T. M. and Brown, R. E. Simulating wind turbine interactions using the vorticity transport model. AIAA-2009-838. In Proceedings of the 47th AIAA Aerospace Sciences Meeting and Exhibit/ASME Wind Energy Symposium, Orlando, January 2009.

18. Scheurich, F., Fletcher, T. M. and Brown R. E. Simulating the aerodynamic performance and wake dynamics of a vertical-axis wind turbine. Wind Energy, in press. Published online in advance of print (4 June 2010). DOI: 10.1002/we.409

19. Strickland, J. H., Smith, T., Sun, K. A vortex model of the Darrieus turbine: an analytical and experimental study. Sandia Technical Report SAND81-7017, 1981. 


\section{Nomenclature}

A swept area

$A R \quad$ aspect ratio, $b / c$

$b \quad$ blade span

$c \quad$ chord length

$C_{n} \quad$ sectional normal force coefficient, $F_{n} / \frac{1}{2} \rho c V_{\infty}^{2}$

$C_{t} \quad$ sectional tangential force coefficient, $F_{t} / \frac{1}{2} \rho c V_{\infty}^{2}$

$C_{Q} \quad$ torque coefficient, $T / \frac{1}{2} \rho A V_{\infty}^{2} R$

$\Delta C_{Q} \quad$ unsteady component of the torque coefficient, $C_{Q}-C_{Q \text {,mean }}$

$F_{n} \quad$ sectional force acting normal to the blade chord

$F_{N} \quad$ force acting normal to the blade chord

$F_{n}^{*} \quad$ sectional non-dimensional normal force, $F_{n} / \frac{1}{2} \rho c V_{\infty}^{2}$

$F_{N}^{*} \quad$ non-dimensional normal force, $F_{N} / \frac{1}{2} \rho b c V_{\infty}^{2}$

$F_{t} \quad$ sectional force acting tangential to the blade chord

$F_{T} \quad$ force acting tangential to the blade chord

$F_{t}^{*} \quad$ sectional non-dimensional tangential force, $F_{t} / \frac{1}{2} \rho c V_{\infty}^{2}$

$F_{T}^{*} \quad$ non-dimensional tangential force, $F_{T} / \frac{1}{2} \rho b c V_{\infty}^{2}$

$F_{X, \text { Rotor }} \mathrm{x}$-component of force on the rotor (streamwise drag force)

$F_{X, \text { Rotor }}^{*}$ non-dimensional streamwise drag force, $F_{X, \text { Rotor }} / \frac{1}{2} \rho A V_{\infty}^{2}$

$F_{Y, \text { Rotor }} \quad \mathrm{y}$-component of force on the rotor (crosswind side force)

$F_{Y, \text { Rotor }}^{*}$ non-dimensional crosswind side force, $F_{Y, \text { Rotor }} / \frac{1}{2} \rho A V_{\infty}^{2}$

$F_{Z, \text { Rotor }}$ Z-component of force on the rotor (axial force)

$F_{Z, \text { Rotor }}^{*}$ non-dimensional axial force, $F_{Z, \text { Rotor }} / \frac{1}{2} \rho A V_{\infty}^{2}$

$H \quad$ turbine height

$Q^{*} \quad$ sectional non-dimensional torque, $F_{t}^{*} r / R$

$r=r(b)$ sectional radius

$R \quad$ radius of the rotor at blade mid-span

$R e \quad$ blade (rotational) Reynolds number, $R e=\Omega R c / \nu$

$S \quad$ vorticity source

$T \quad$ torque

$u \quad$ flow velocity

$u_{b} \quad$ flow velocity relative to the blade

$V_{\infty} \quad$ wind speed/ free stream velocity

$\alpha \quad$ angle of attack

$\lambda \quad$ reference tip speed ratio, $\Omega R / V_{\infty}$

$\nu \quad$ kinematic viscosity

$\psi \quad$ azimuth angle

$\rho \quad$ density

$\omega \quad$ vorticity

$\omega_{b} \quad$ bound vorticity

$\Omega \quad$ angular velocity of the rotor 
Table 1: Geometric properties of the investigated turbine configurations.

straight-bladed curved-bladed helically twisted

$\begin{array}{llll}H / R & 3.00 & 3.00 & 3.00 \\ r / R \text { at mid-span } & 1.00 & 1.00 & 1.00 \\ r / R \text { at blade tips } & 1.00 & 0.33 & 0.73 \\ \text { helical twist of blade tips } * & 0.00 & 0.00 & \pm 82^{\circ}\end{array}$

* i.e. azimuth positions of blade tips compared to mid-span.

Table 2: Rotor parameters of the straight-bladed turbine.

Number of blades

Aerofoil section NACA 0015

Rotational Reynolds number at blade mid-span 800,000

Chord-to-radius ratio at mid-span

Aspect ratio

Tip speed ratio

Table 3: Torque coefficients and rotor forces.

\begin{tabular}{lccc}
\hline \hline & & & \\
& straight-bladed & curved-bladed & helically twisted \\
$C_{Q, \text { mean }}$ & 0.021 & 0.046 & 0.076 \\
$F_{X, \text { Rotor,mean }}^{*}$ & 1.265 & 1.307 & 1.2751 \\
$F_{Y, \text { Rotor,mean }}^{*}$ & -0.147 & -0.252 & -0.189 \\
$F_{Z, \text { Rotor,mean }}^{*}$ & 0.000 & -0.003 & -0.034 \\
& & & \\
\hline \hline
\end{tabular}




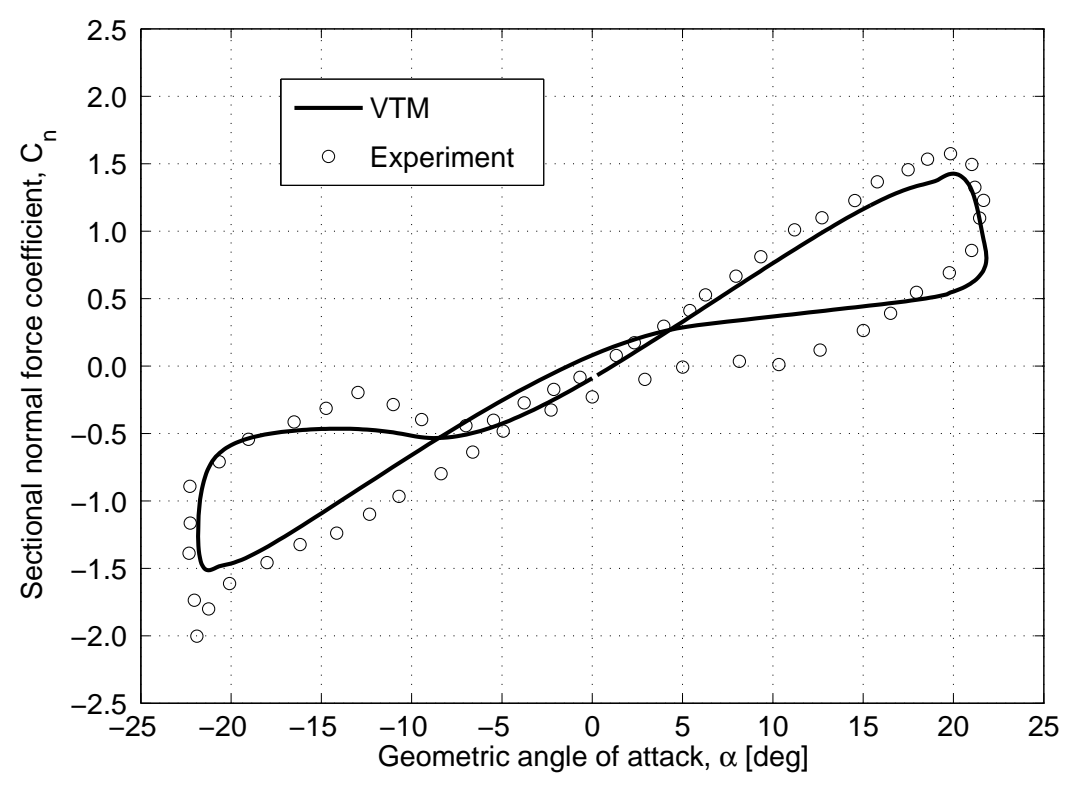

(a) normal force coefficient

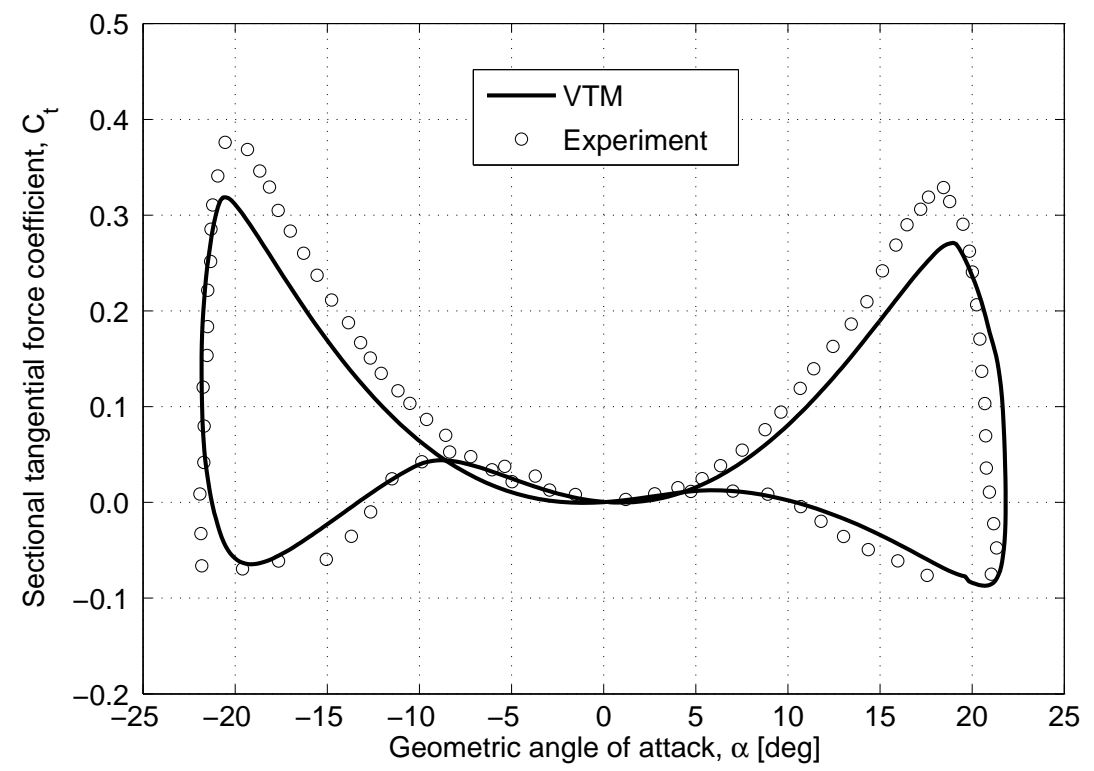

(b) tangential force coef ficient

Figure 1: VTM-predicted normal and tangential force coefficients during a dynamic stall test of a NACA 0015 aerofoil at $R e=800,000$ in comparison to experimental measurements made by Angell, Musgrove and Galbraith [12]. A skewed sinusoidal variation of angle of attack (with amplitude $21.80^{\circ}$ ) was used in order to represent vertical-axis wind turbine conditions at $\lambda=2.70$. 


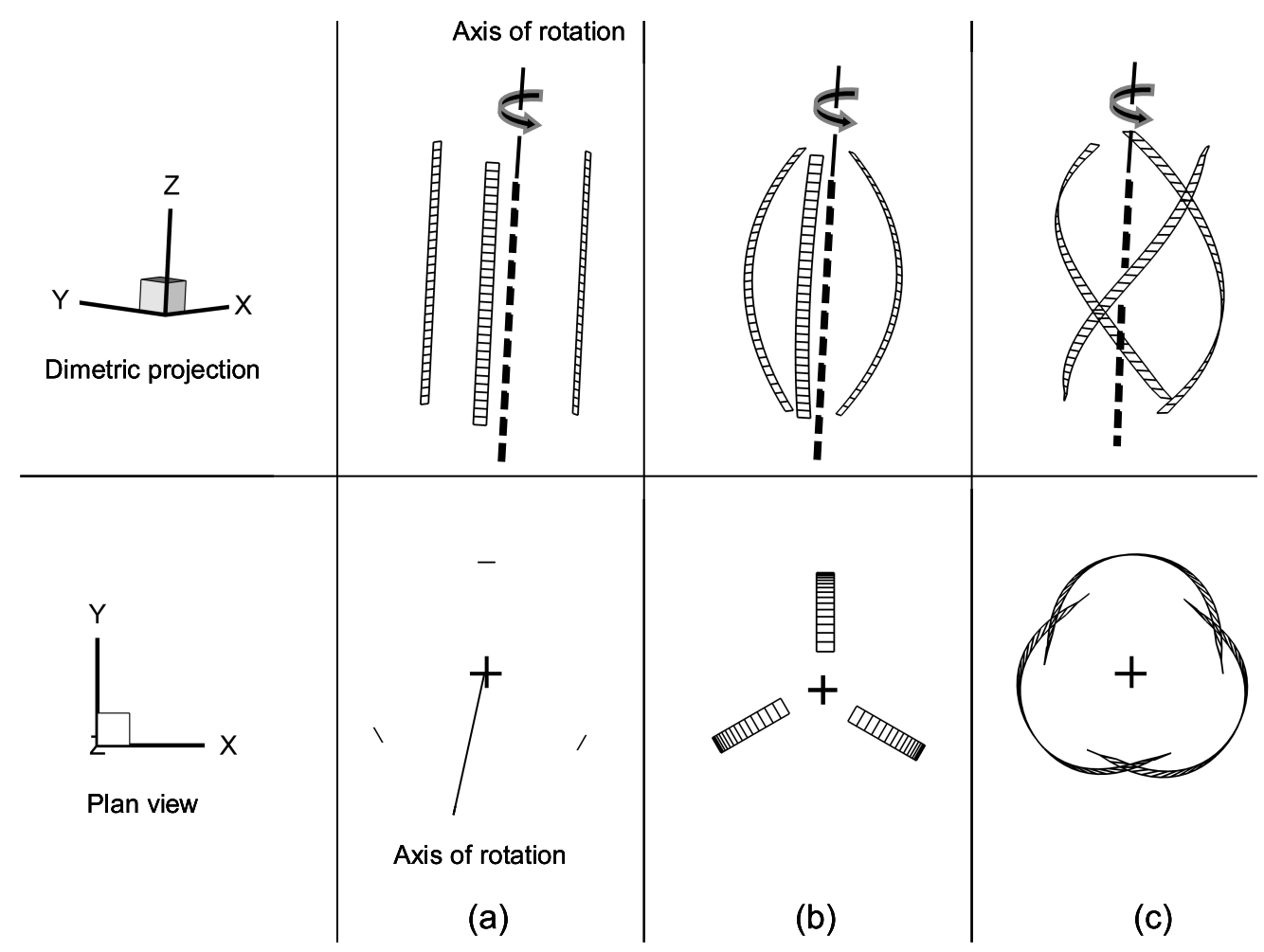

Figure 2: Geometry of the vertical-axis wind turbines with straight (a), curved (b), and helically twisted blades (c). 


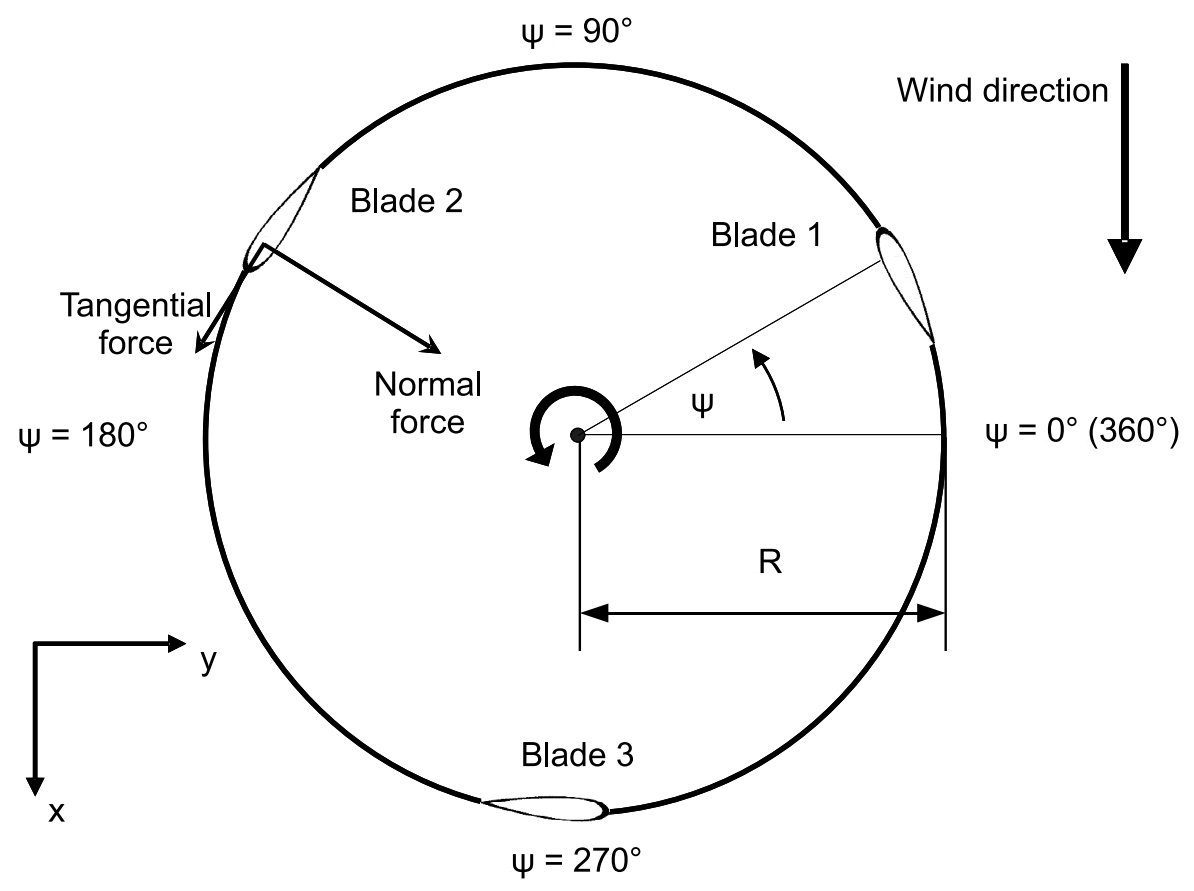

Figure 3: Diagram showing the wind direction, the definition of rotor azimuth and direction of positive normal and tangential forces, as well as the relative positions of the rotor blades. 


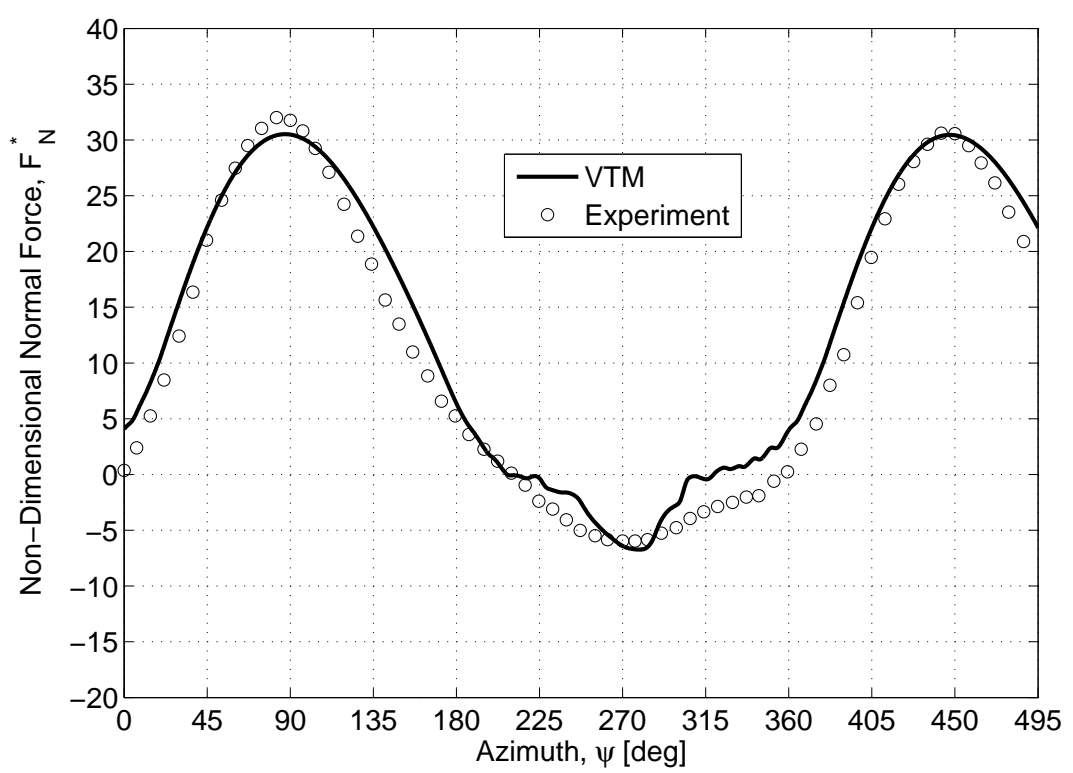

(a) Normal force

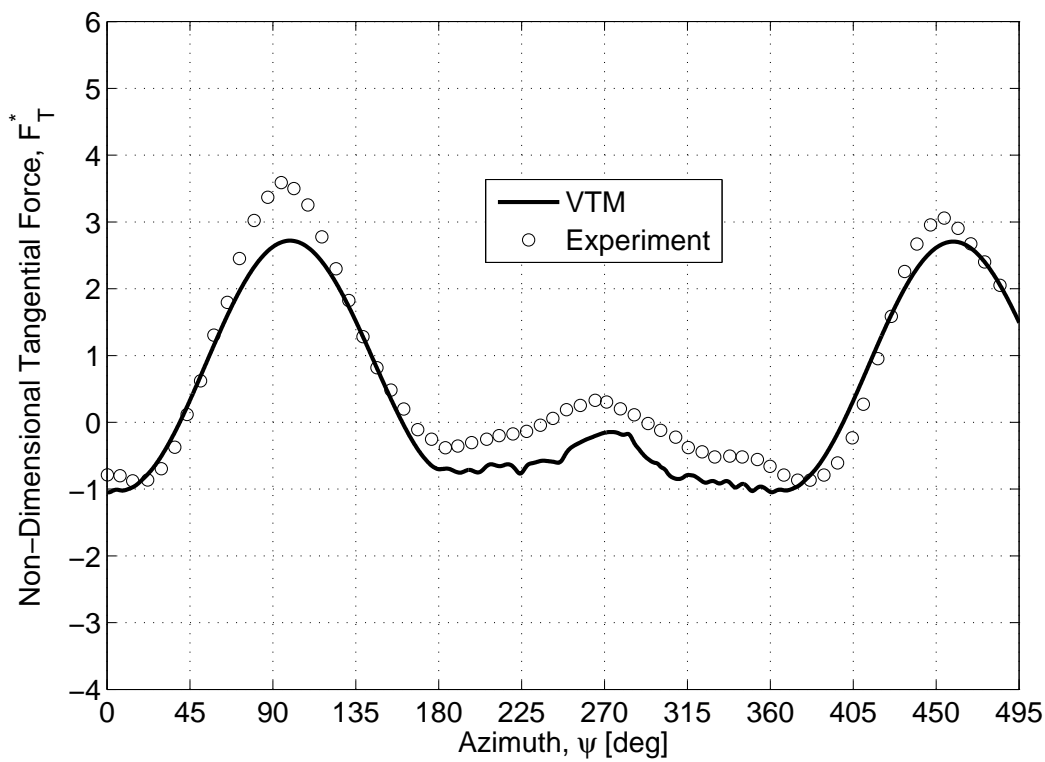

(b) Tangential force

Figure 4: Comparison of VTM-predicted non-dimensional forces with experimental measurements made by Strickland, Smith and Sun [19]. Note: the sign convention for the normal force is opposite to that used in Ref. 19. 


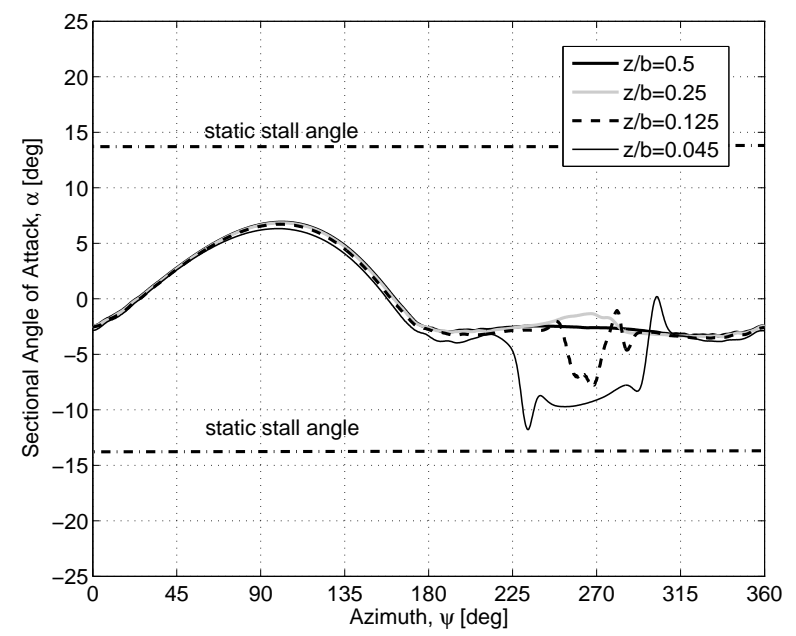

(a) Angle of attack

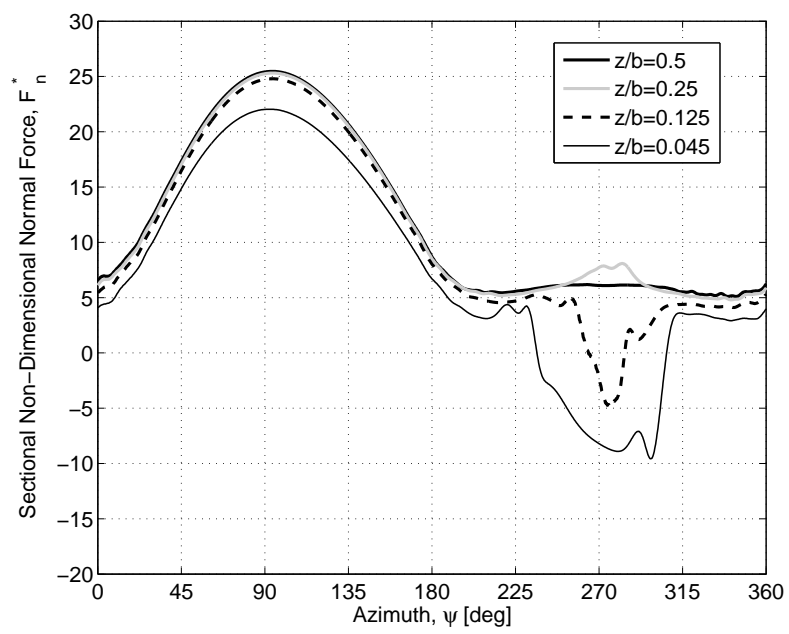

(c) Normal force

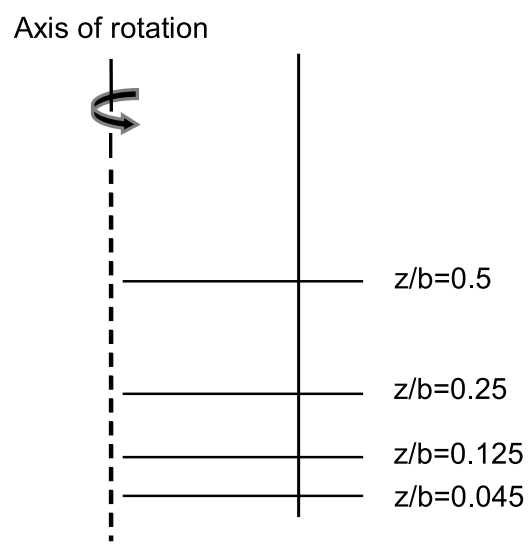

(b) Location

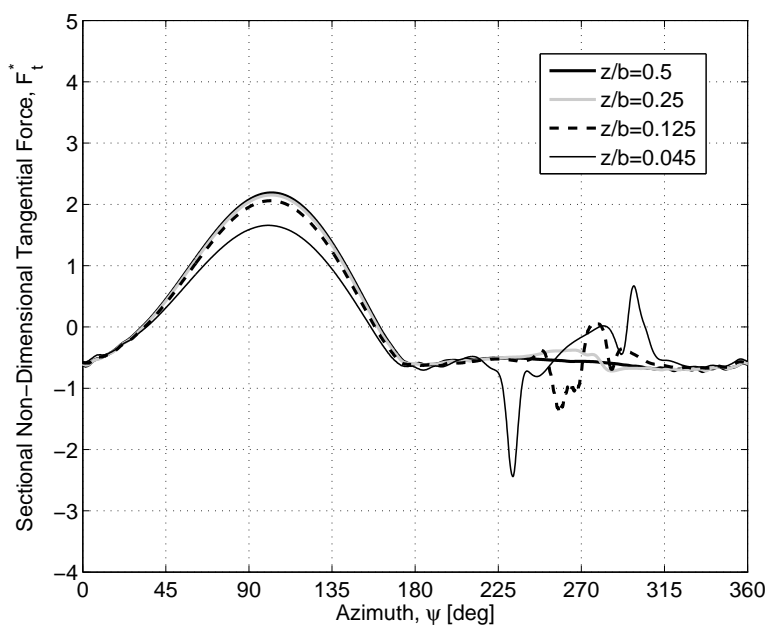

(d) Tangential force

Figure 5: VTM-predicted forces and angle of attack distribution over one complete rotor revolution at four different spanwise stations along the straight-bladed turbine (configuration 'a'). 


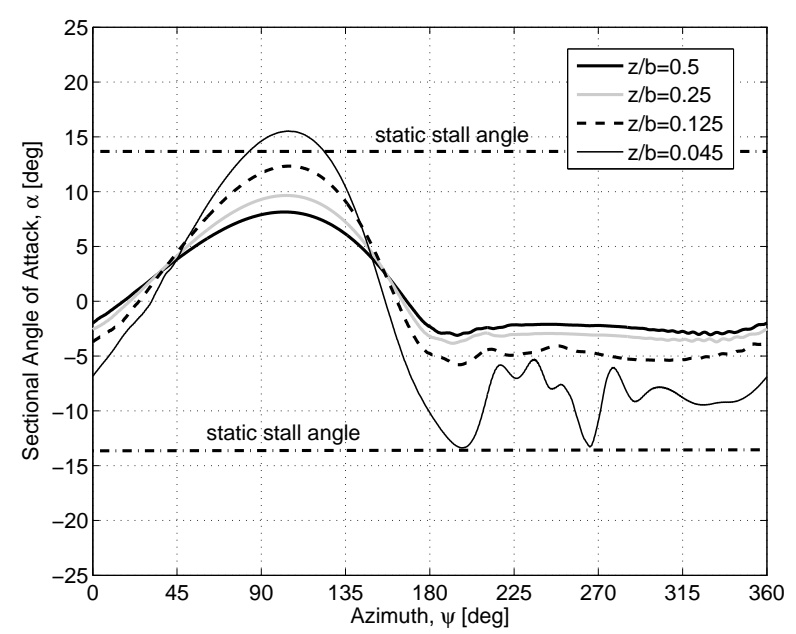

(a) Angle of attack

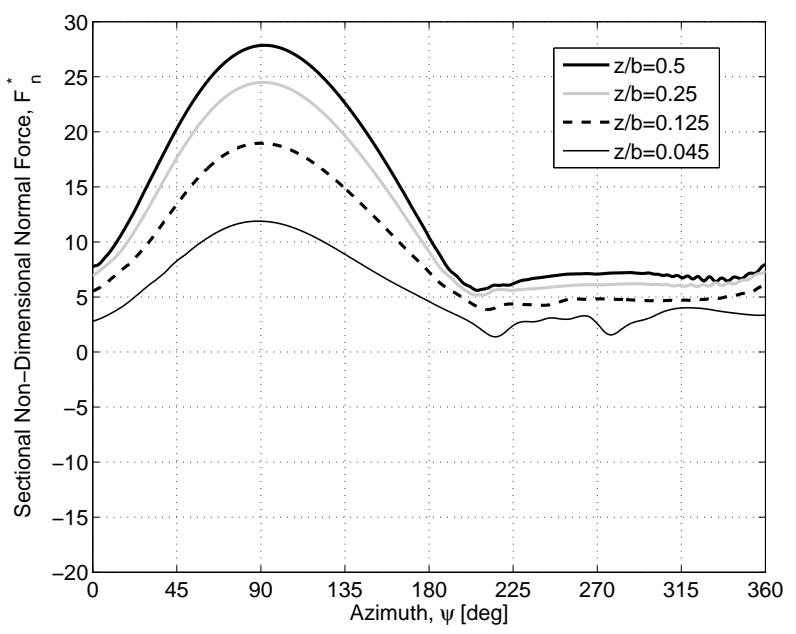

(c) Normal force

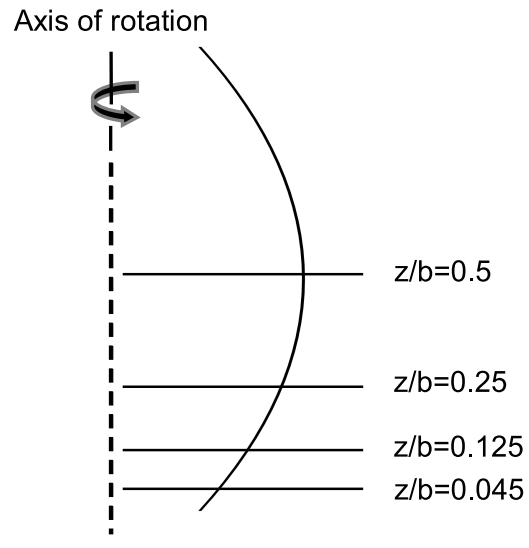

(b) Location

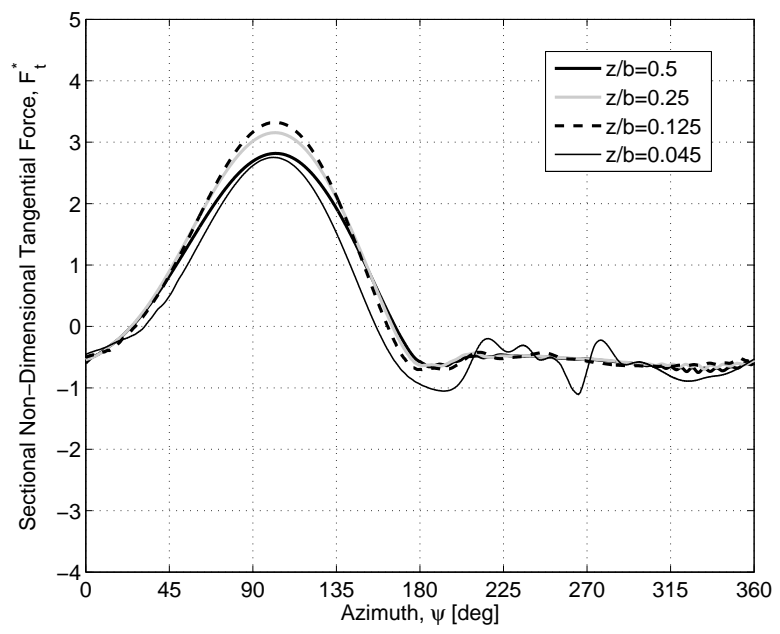

(d) Tangential force

Figure 6: VTM-predicted forces and angle of attack distribution over one complete rotor revolution at four different spanwise stations along the curved-bladed turbine (configuration 'b'). 


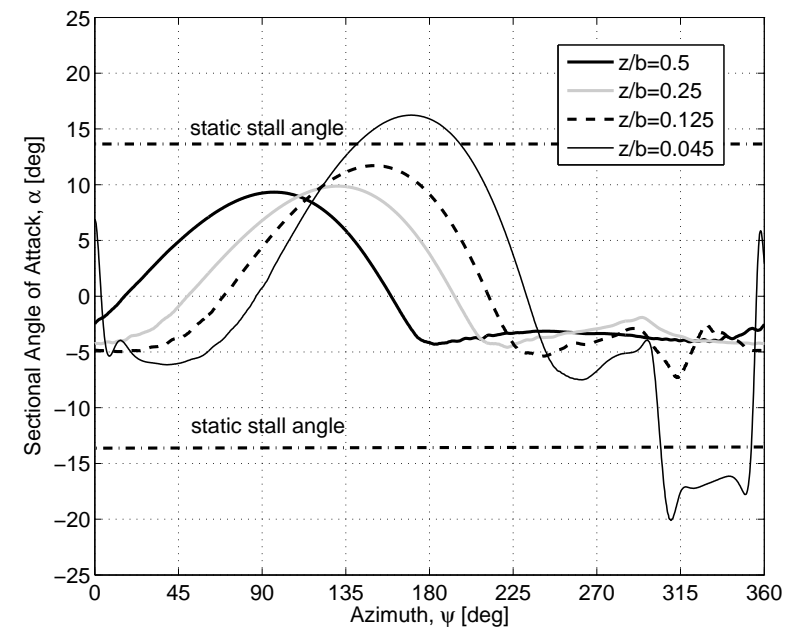

(a) Angle of attack

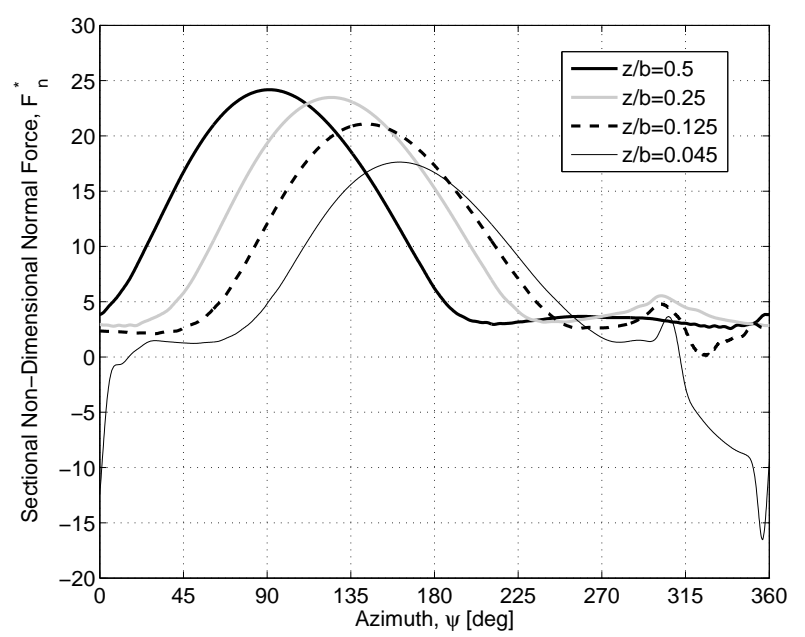

(c) Normal force

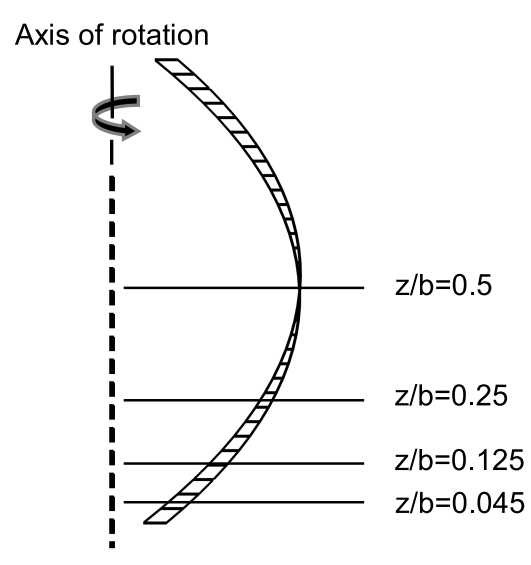

(b) Location

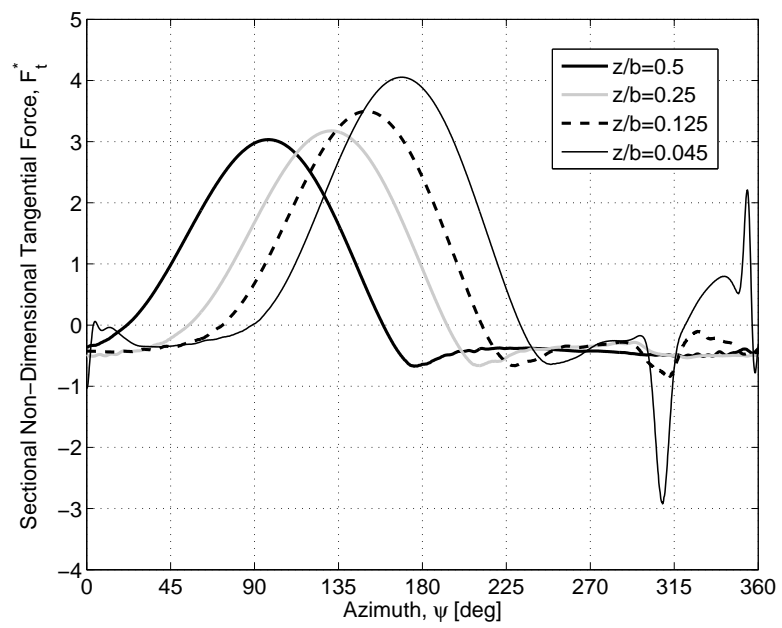

(d) Tangential force

Figure 7: VTM-predicted forces and angle of attack distribution over one complete rotor revolution at four different spanwise stations along the turbine with helically twisted blades (configuration ' $\mathrm{c}$ '). The azimuth $\psi$ is with respect to the section at the mid-span of the reference blade ('blade 1'). 


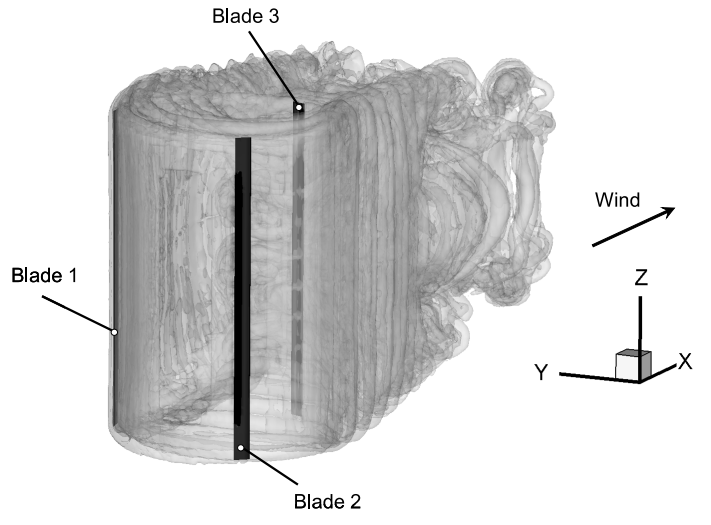

(a)
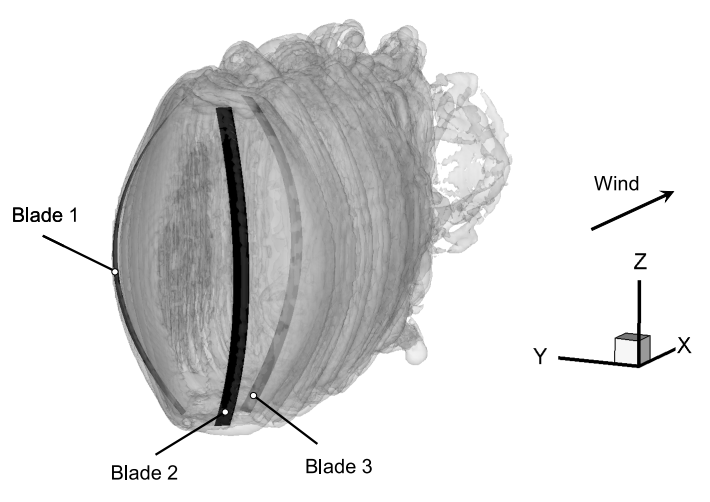

(c)

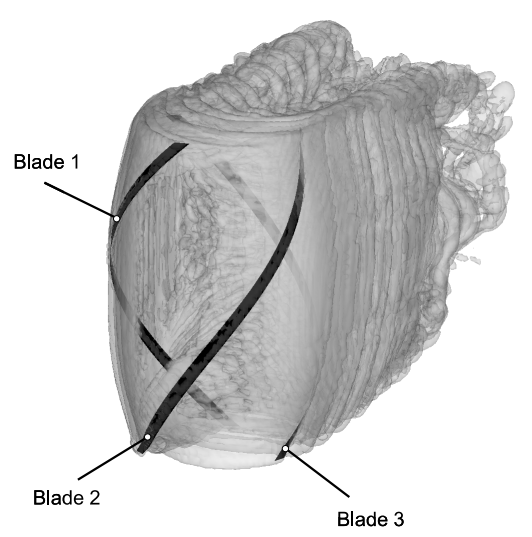

(e)

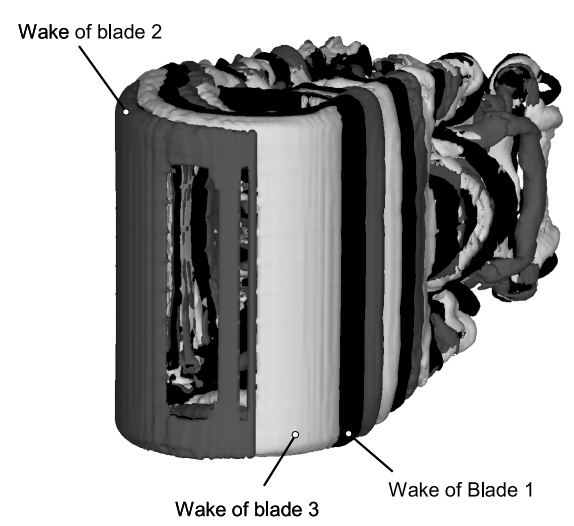

(b)

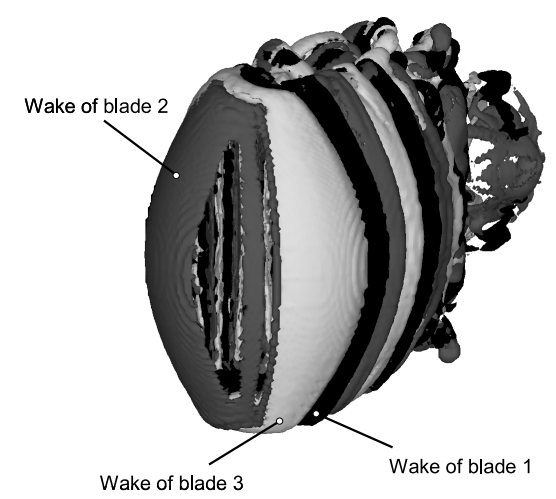

(d)

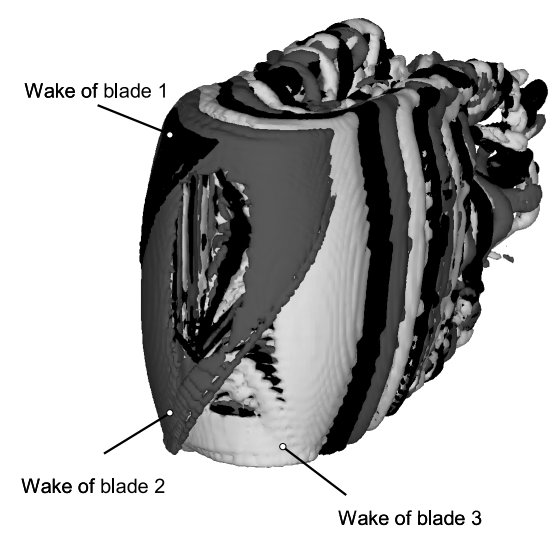

(f)

Figure 8: Three-dimensional graphical representation of the computed flow fields surrounding the turbine configurations with straight blades (a,b), curved blades (c,d), and helically twisted blades $(e, f)$. The wake geometry is visualized by rendering a surface on which the vorticity has constant magnitude. The sub-figures on the left show the entire vorticity field that is developed by the turbine, whereas the subfigures on the right show the vorticity that is developed by each individual blade. 


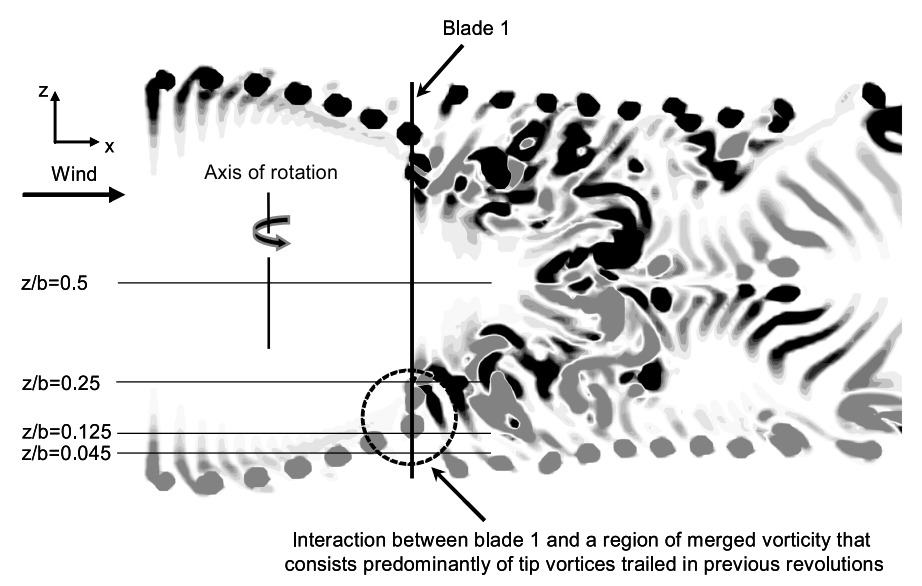

(a) straight-bladed turbine

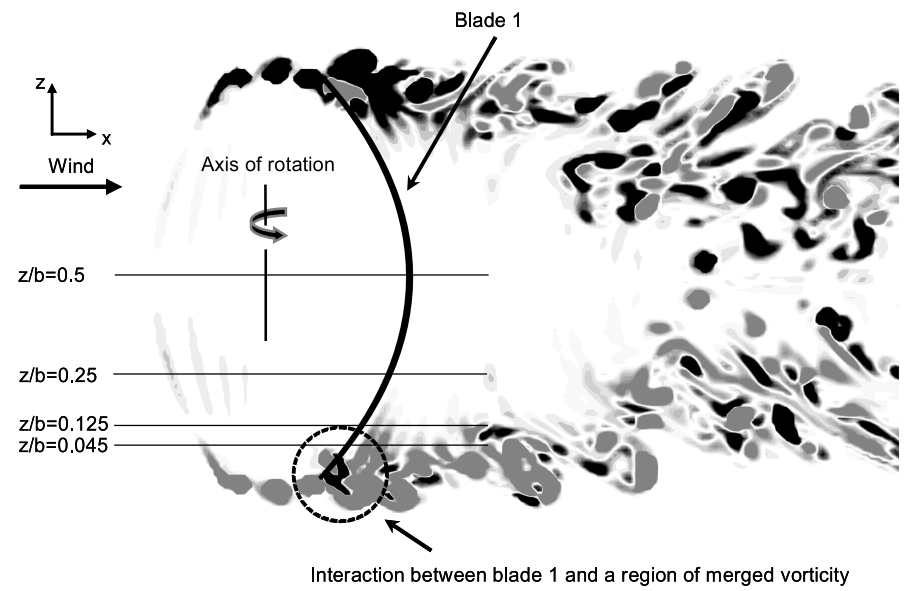

(b) curved-bladed turbine

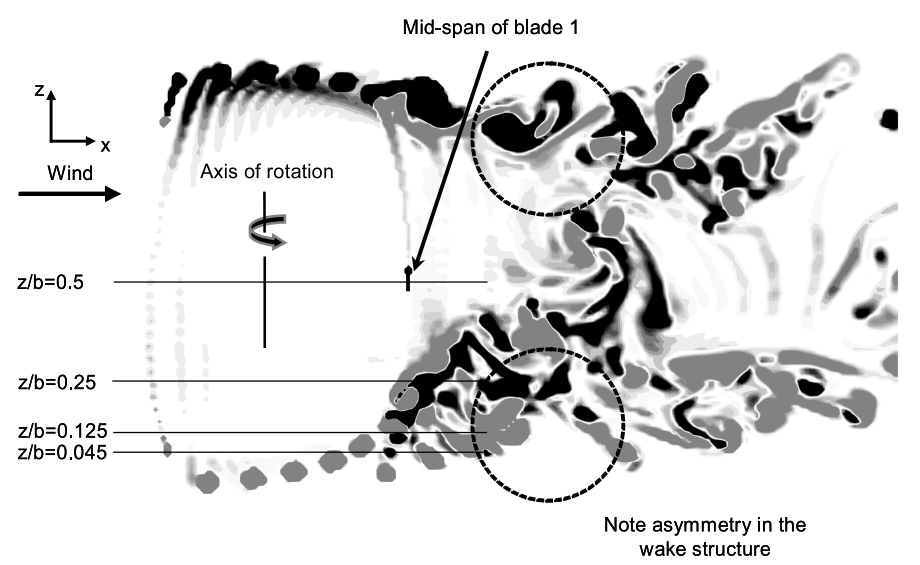

(c) turbine with helically twisted blades

Figure 9: Computed vorticity fields surrounding the three turbine configurations that were investigated, represented using contours of vorticity on a vertical plane that contains the axis of rotation of the turbine, and that is aligned with the wind direction. Blade 1 is located at azimuth $\psi=270^{\circ}$. 


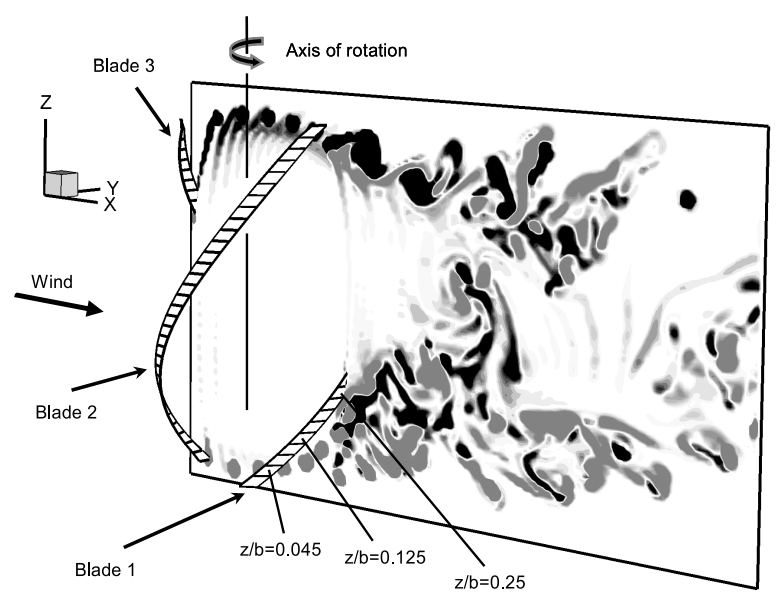

(a) mid - span of blade 1 at $\psi=300^{\circ}$

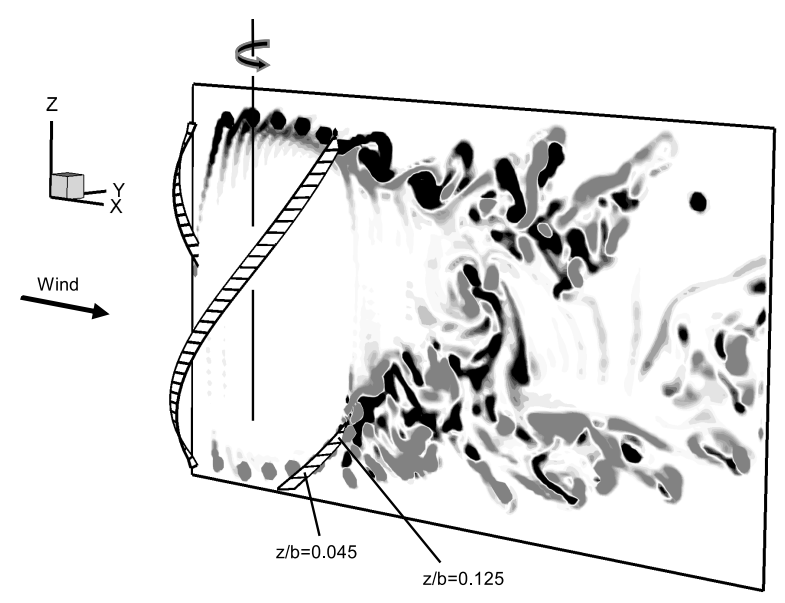

(b) mid - span of blade 1 at $\psi=320^{\circ}$

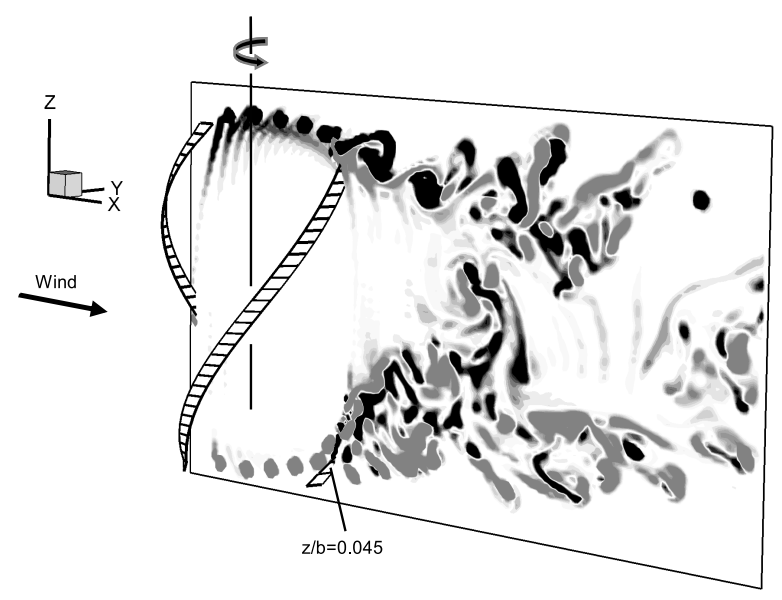

(c) mid-span of blade 1 at $\psi=340^{\circ}$

Figure 10: Computed vorticity field surrounding the turbine with helically twisted blades. Visualization by contours of the vorticity component that is perpendicular to a vertical plane that contains the axis of rotation of the turbine, and that is aligned with the wind direction. 


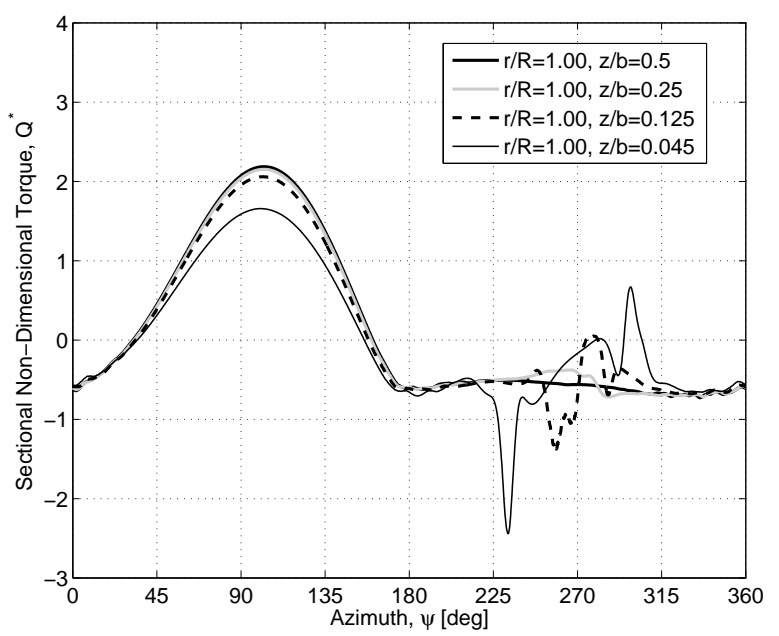

(a) straight - bladed turbine

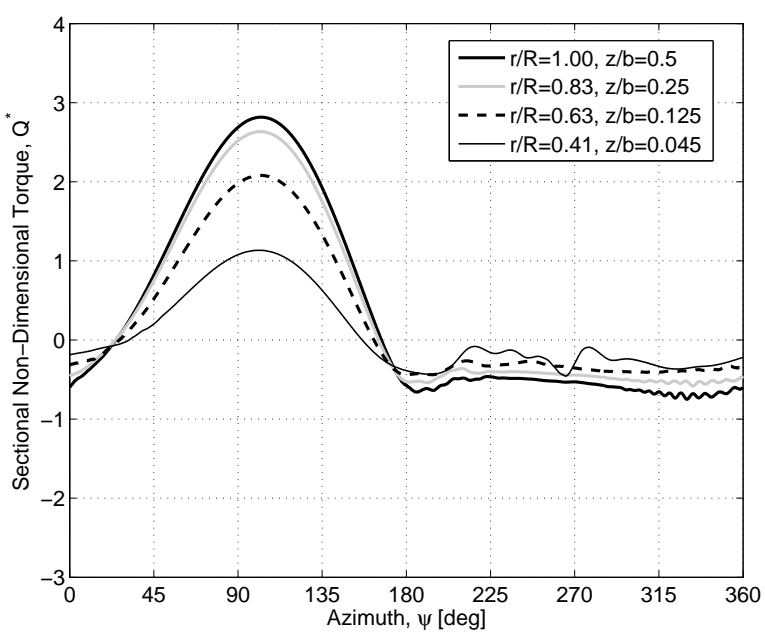

(b) curved-bladed turbine

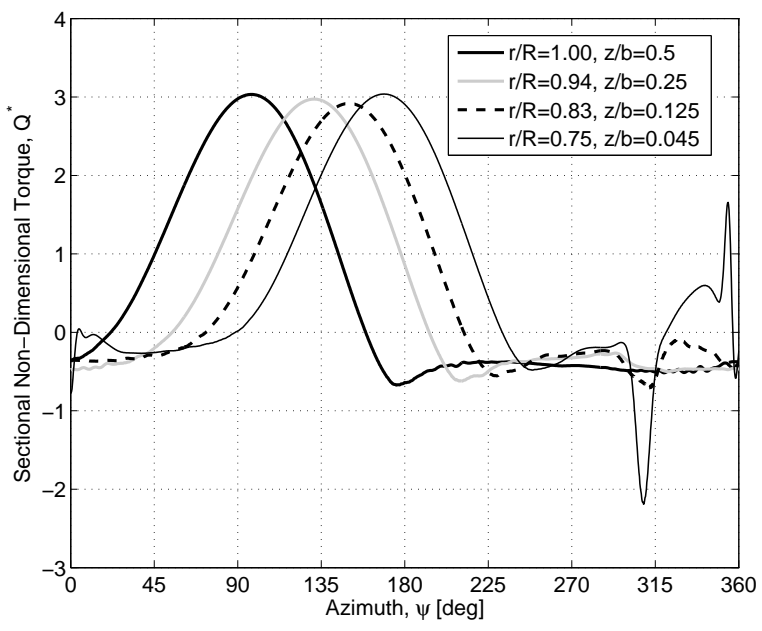

(c) turbine with helically twisted blades

Figure 11: VTM-predicted sectional non-dimensional torque at four blade sections for the three turbine configurations that were investigated. 


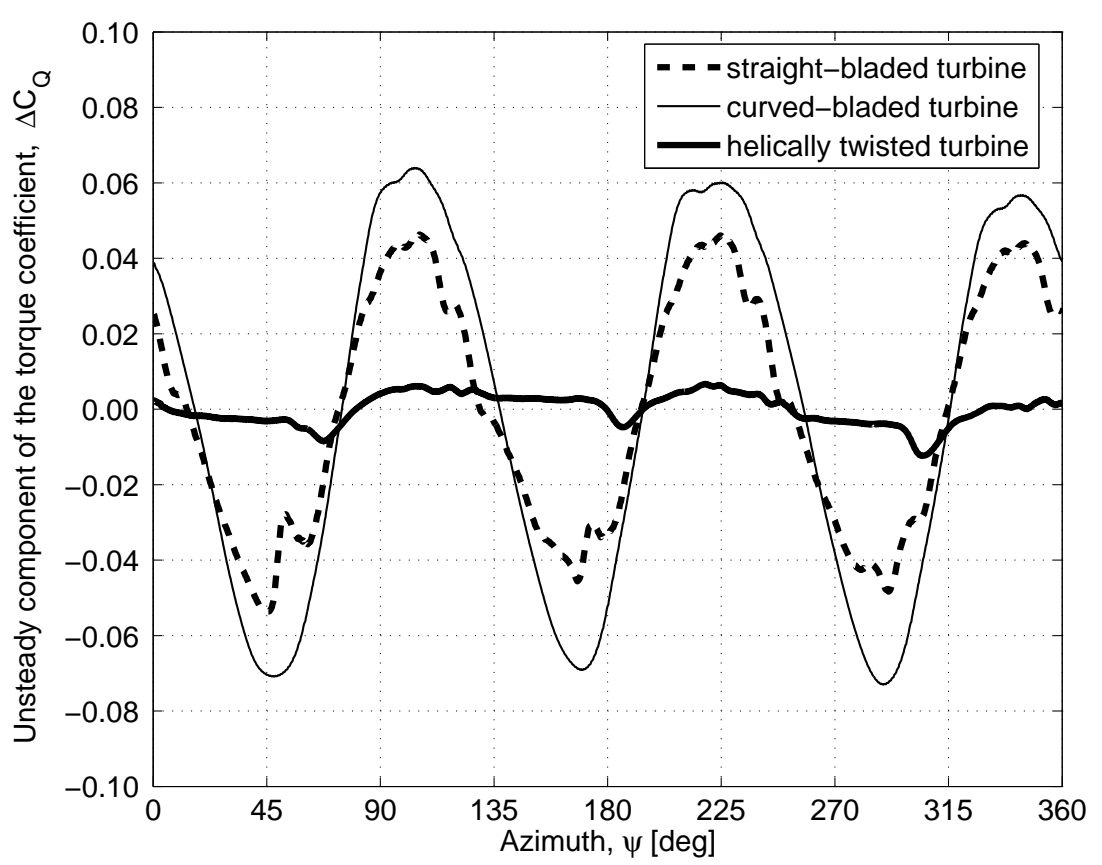

Figure 12: VTM-predicted variation of torque coefficient with azimuth angle for the three turbine configurations that were investigated. 


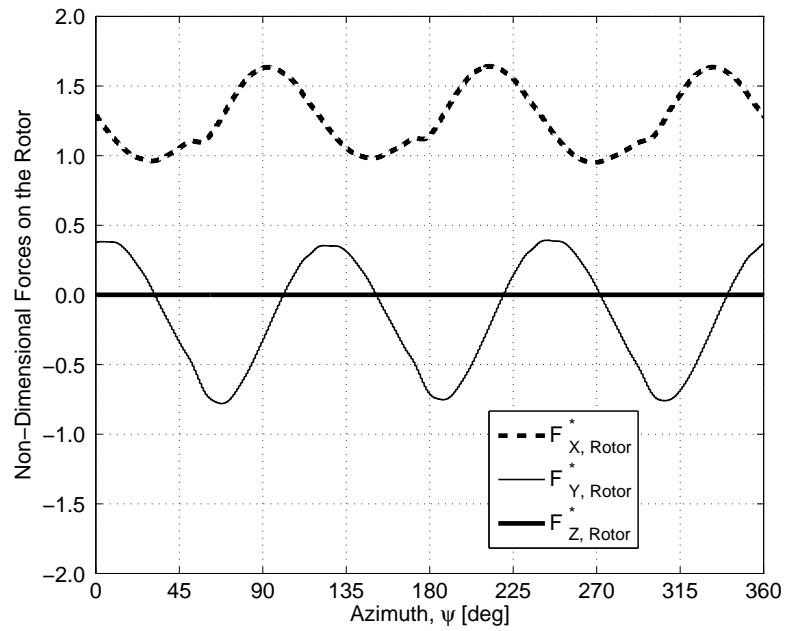

(a) straight - bladed turbine

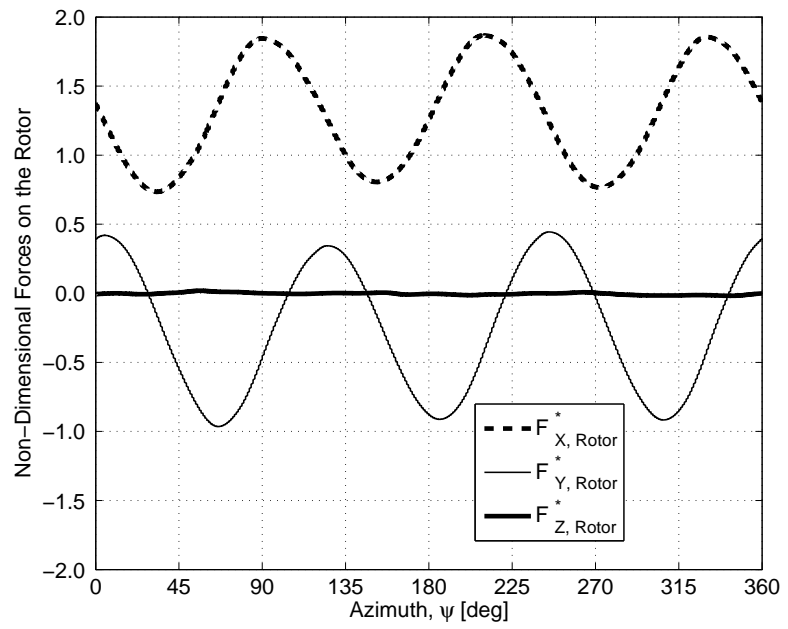

(b) curved-bladed turbine

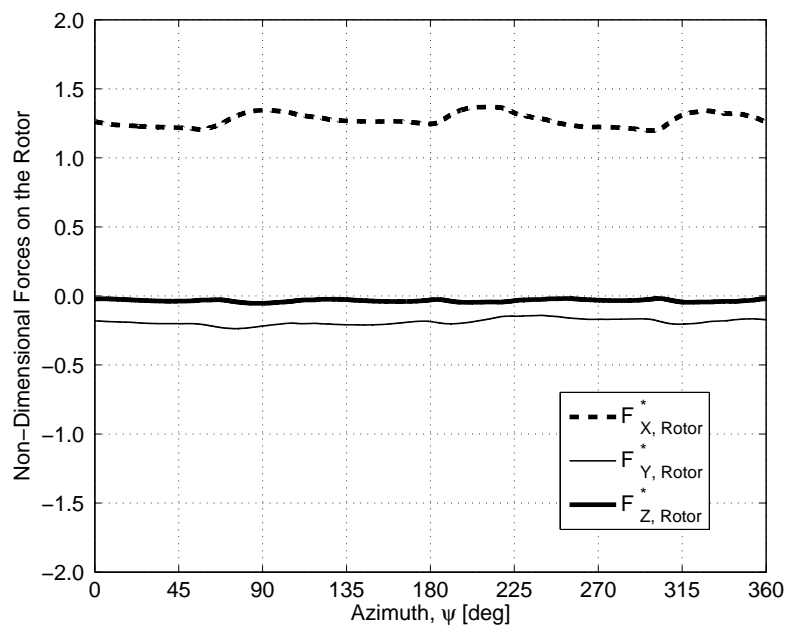

(c) turbine with helically twisted blades

Figure 13: VTM-predicted forces on the rotor for the three turbine configurations that were investigated. 